\title{
Entropies of general nonextreme stationary axisymmetric black hole: statistical mechanics and thermodynamics *
}

\author{
Jiliang Jing ${ }^{a b} \quad$ Mu-Lin Yan ${ }^{a}$ \\ a)Department of Astronomy and Applied Physics, University of Science and Technology of China, \\ Hefei, Anhui 230026, P. R. China; \\ b) Physics Department and Institute of Physics, Hunan Normal University, \\ Changsha, Hunan 410081, P. R. China
}

\begin{abstract}
Starting from metric of general nonextreme stationary axisymmetric black hole in four-dimensional spacetime, both statistical-mechanical and thermodynamical entropies are studied. First, by means of the "brick wall" model in which the Dirichlet condition is replaced by a scattering ansatz for the field functions at the horizon and with the Pauli-Villars regularization scheme, an expression for the statistical-mechanical entropy arising from the nonminimally coupled scalar fields is obtained. Then, by using the conical singularity method Mann and Solodukhin's result for the Kerr-Newman black hole (Phys. Rev. D54, 3932(1996)) is extended to the general stationary black hole and nonminimally coupled scalar fields. We last show by comparing the two results that the statistical-mechanical entropy and one-loop correction to the thermodynamical entropy are equivalent for coupling $\xi \leq 0$. After renormalization, a relation between the two entropies is given.
\end{abstract}

PACS numbers: 04.70.Dy, 04.62.+V, 97.60.Lf.

*This work was partially supported by the National Nature Science Foundation of China under Grant No. 19975018, and Nature Science Foundation of Hunan Province 


\section{INTRODUCTION}

There are two definitions of entropy of fields on the black hole exterior. One definition is based on the covariant Euclidean formulation, another one is canonical. The thermodynamical entropy of a black hole is related to the covariant Euclidean free energy $F^{E}[g, \beta]=\beta^{-1} W[g, \beta]$ [1], where $\beta$ is the inverse temperature. Function $W[g, \beta]$ is given on Euclidean manifolds with the period $\beta$ in Euclidean time $\tau$. An alternative approach to calculate $F^{E}$ is the conical singularities method [2]- [11]. The statistical-mechanical entropy can be derived from the canonical formulation [1]. The corresponding free energy, $F^{C}$, can be defined in term of the one-particle spectrum. One of the ways to calculate $F^{C}$ is "brick wall" model (BWM) proposed by 't Hooft [12]. In the model, in order to eliminate divergence which appears due to the infinite growth of the density of states close to the horizon, 't Hooft introduces a "brick wall" cutoff: a fixed boundary near the event horizon within the quantum field does not propagate and the Dirichlet boundary condition is imposed on the boundary. In order to get the correct $\xi$ (coupling constant) dependence for the statisticalmechanical entropy and obtained the correspondence with the conical singularity method, Solodukhin [13] introduced a scattering ansatz, i.e., the field function near the event horizon that describes scattering by the hole with some nontrivial change of phase.

For ordinary thermodynamical systems it is well known that the thermodynamical and the statistical-mechanical entropies are exactly the same. For the black holes, the study of the two entropies has attracted much attention recently [1] [12]- [31]. The study was motivated by attempts to explain the entropy of black holes as the statistical-mechanical entropy of quantum fields propagating near the event horizon. In Ref. 28] Solodukhin demonstrated in the two-dimensional example that the thermodynamical entropy of a black hole coincides with its statistical-mechanical one. In Ref. [13] Solodukhin calculated the statistical-mechanical entropy for a scalar filed with nonminimally coupling in the fourdimensional static black hole spacetime and found that for $\xi \leq 0$ the result agrees with one-loop correction to the thermodynamical entropy [6]. Frolov and Fursaev [1] reviewed studies of the relation between the thermodynamic entropy and the statistical-mechanical entropy of black holes. They showed that the covariant Euclidean free energy $F^{E}$ and the canonical free energy $F^{C}$ are equivalent when ones use the ultraviolet regularization [1] for the general static black holes.

Mann and Solodukhin expected in Ref. [11] that study of the two entropies for the stationary axisymmetric black hole should provide us with better understanding of the relationship between the different entropies. It is general opinion that the study of the quantum entropies for the stationary axisymmetric black hole in four-dimensional spacetime is an interesting topic and should be investigated deeply. Therefore, much effort has also been contributed the research in the last years [11] [20] [16] [21]. Mann and Solodukhin [11] studied thermodynamical entropy of the Kerr-Newman black hole due to a minimally coupled scalar field by using the conical singularities method. Cognola [20], through the Euclidean path integral and using a heat kernel and $\zeta$-function regularization scheme, studied the oneloop contribution to the entropy for a scalar field in the Kerr black hole (he pointed that the result is valid also for the Kerr-Newman black hole). Unfortunately, the result is in contrast with the corresponding one obtained by the conical singularities [11. 
Recently, we [32] studied the statistical-mechanical entropy by using the BWM and with the Pauli-Villars regularization scheme. We showed that one-loop correction to the thermodynamical entropy and the statistical-mechanical entropy due to the scalar quantum fields are equivalent for the Kerr-Newman and the Einstein-Maxwell dilaton-axion black holes. However, at the moment the relation between two entropies for general nonextreme stationary axisymmetric black hole still remains open. The aim of this paper is to settle the question for nonextreme case.

The paper is organized as follows: In Sec. II, by using the BWM [13] we deduce a formula of statistical entropy arising from nonminimally coupled scalar fields in the general nonextreme stationary axisymmetric black hole spacetime. In sec. III, by means of the conical singularities method we extend Mann and Solodukhin's result in Ref. [11 to the nonminimally coupled scalar fields and the general stationary axisymmetric black hole. Two results are then compared and a summary is presented in last section. Some calculations are given in Appendices.

\section{STATISTICAL-MECHANICAL ENTROPY OF GENERAL NONEXTREME STATIONARY AXISYMMETRIC BLACK HOLES}

The metric for the general stationary axisymmetric black hole in Boyer-Lindquist coordinates can be expressed as

$$
d s^{2}=g_{t t} d t^{2}+g_{r r} d r^{2}+g_{t \varphi} d t d \varphi+g_{\theta \theta} d \theta^{2}+g_{\varphi \varphi} d \varphi^{2}
$$

where $g_{t t}, g_{r r}, g_{t \varphi}, g_{\theta \theta}$ and $g_{\varphi \varphi}$ are functions of the coordinates $r$ and $\theta$ only. For the nonextreme case we have relations (see [32] for details)

$$
\begin{aligned}
\left(g_{t t}-\frac{g_{t \varphi}^{2}}{g_{\varphi \varphi}}\right) & =G_{1}(r, \theta)\left(r-r_{H}\right), \\
g^{r r} & =G_{2}(r, \theta)\left(r-r_{H}\right), \\
g_{r r}\left(g_{t t}-\frac{g_{t \varphi}^{2}}{g_{\varphi \varphi}}\right) & =\frac{G_{1}(r, \theta)}{G_{2}(r, \theta)} \equiv-f(r, \theta),
\end{aligned}
$$

where $r_{H}$ represents event horizon, $G_{1}(r, \theta), G_{2}(r, \theta)$ and $f(r, \theta)$ are regular functions on and outside the event horizon.

We now try to find a general statistical-mechanical entropy expression for the spacetime (2.1). We first seek quantization condition by using the motion equation of the scalar

field and introducing the scattering boundary condition, and then use the condition to calculate free energy. The statistical-mechanical entropy is obtained by the variation of the corresponding free energy with respect to the inverse Hawking temperature.

Using the a WKB approximation with

$$
\phi=\exp [-i E t+i m \phi+i W(r, \theta)]=\exp [-i E t+i m \phi] \psi(r, \theta),
$$

and substituting the metric (2.1) into the motion equation of the scalar field with mass $\mu$ and arbitrary coupled to the scalar curvature $R$ 


$$
\frac{1}{\sqrt{-g}} \partial_{\mu}\left(\sqrt{-g} g^{\mu \nu} \partial_{\nu} \phi\right)-\left(\mu^{2}+\xi R\right) \phi=0
$$

We know from discussion in Ref. [32] that the function $W(r, \theta)$ can be expressed as

$$
W(r, \theta)= \pm \int^{r} \sqrt{\frac{-g_{r r} g_{\varphi \varphi}}{g_{t t} g_{\varphi \varphi}-g_{t \varphi}^{2}}} K(r, \theta) d r+c(\theta),
$$

where

$$
K(r, \theta)=\sqrt{(E-\Omega m)^{2}+\left(g_{t t}-\frac{g_{t \varphi}^{2}}{g_{\varphi \varphi}}\right)\left(\frac{m^{2}}{g_{\varphi \varphi}}+\frac{p_{\theta}^{2}}{g_{\theta \theta}}+M^{2}(r, \theta)\right)} .
$$

Then, the function $\psi(r, \theta)$ in (2.5) is given by

$$
\begin{aligned}
\psi(r, \theta)= & \exp \left[i \int^{r} \sqrt{\frac{-g_{r r} g_{\varphi \varphi}}{g_{t t} g_{\varphi \varphi}-g_{t \varphi}^{2}}} K(r, \theta) d r\right] \\
& +A \exp \left[-i \int^{r} \sqrt{\frac{-g_{r r} g_{\varphi \varphi}}{g_{t t} g_{\varphi \varphi}-g_{t \varphi}^{2}}} K(r, \theta) d r\right],
\end{aligned}
$$

where the constant $A$ is to be determined with the boundary conditions. In Eq. (2.9) the amplitude is a slowly varying function and is omitted in writing. At the boundary $\Sigma_{h}$ staying at a small distance $h$ from the event horizon $\Sigma$, Solodukhin's scattering condition [13] is shown by

$$
\left.\left(n^{\mu} \partial_{\mu} \phi-\xi k \phi\right)\right|_{\Sigma_{h}}=0
$$

where $n^{\mu}$ is vector normal to $\Sigma_{h}$ and $k$ is extrinsic curvature of $\Sigma_{h}$. For the stationary axisymmetric black hole (2.1), after setting

$$
n_{\mu}=\left(0, \sqrt{g_{r r}}, 0,0\right)
$$

we find the extrinsic curvature can be written as [33]

$$
\begin{aligned}
k= & \frac{\sqrt{g_{r r}}}{2 g_{r r} g_{\theta \theta}\left(g_{t t} g_{\varphi \varphi}-g_{t \varphi}^{2}\right)}\left[\left(g_{t t} g_{\varphi \varphi}-g_{t \varphi}^{2}\right) \frac{\partial g_{\theta \theta}}{\partial r}\right. \\
& \left.+g_{t t} g_{\theta \theta} \frac{\partial g_{\varphi \varphi}}{\partial r}-2 g_{t \varphi} g_{\theta \theta} \frac{\partial g_{t \varphi}}{\partial r}+g_{\varphi \varphi} g_{\theta \theta} \frac{\partial g_{t t}}{\partial r}\right]
\end{aligned}
$$

on the boundary $\Sigma_{h}$ Eq. (2.12) reduces to

$$
k\left(\Sigma_{h}\right) \approx\left[\frac{1}{2 \sqrt{g_{r r}}\left(g_{t t}-\frac{g_{t \varphi}^{2}}{g_{\varphi \varphi}}\right)} \frac{\partial}{\partial r}\left(g_{t t}-\frac{g_{t \varphi}^{2}}{g_{\varphi \varphi}}\right)\right]_{\Sigma_{h}}
$$

From Eqs. (2.9), (2.13) and (2.10) we have 


$$
\left[\sqrt{\frac{-\left(g_{t t}-\frac{g_{t \varphi}^{2}}{g_{\varphi \varphi}}\right)}{g_{r r}}} \frac{\partial \psi(r, \theta)}{\partial r}-\xi^{*} \psi(r, \theta)\right]_{\Sigma_{h}}=0
$$

where $\xi^{*} \equiv \frac{2 \pi \xi}{\beta_{H}}$ and $\beta_{H}$ is the Hawking inverse temperature. Making use of Eq. (2.14) and another boundary condition $\phi=0$, for $r=r_{E}\left(r_{E}<r_{V L S}\right.$, where $r_{V L S}$ is position of the velocity of the light surface [16] [21]), after discussion as Solodukhin [13] we find following quantum condition

$$
2 \int_{r_{H}+h}^{r_{E}} \sqrt{\frac{-g_{r r} g_{\varphi \varphi}}{g_{t t} g_{\varphi \varphi}-g_{t \varphi}^{2}}} K(r, \theta) d r=\nu \eta(K)+\pi \nu+2 \pi n\left(E, m, p_{\theta}, \theta\right)
$$

with

$$
\eta(K)=\tan ^{-1}\left(\frac{2 K(h) \xi^{*}}{K(h)^{2}-\xi^{* 2}}\right), \quad \nu=2 \xi
$$

We can separate $n\left(E, m, p_{\theta}, \theta\right)$ into two parts

$$
n\left(E, m, p_{\theta}, \theta\right)=n_{0}\left(E, m, p_{\theta}, \theta\right)+n_{1}\left(E, m, p_{\theta}, \theta\right) .
$$

From Eqs. (2.15) and (2.16) we find that $n_{0}\left(E, m, p_{\theta}, \theta\right)$ and $n_{1}\left(E, m, p_{\theta}, \theta\right)$ can be respectively expressed as

$$
\begin{gathered}
n_{0}\left(E, m, p_{\theta}\right)=\frac{1}{\pi} \int d \theta \int_{r_{H}+h}^{r_{E}} \sqrt{\frac{-g_{r r} g_{\varphi \varphi}}{g_{t t} g_{\varphi \varphi}-g_{t \varphi}^{2}}} K(r, \theta) d r, \\
n_{1}\left(E, m, p_{\theta}\right)=\frac{-\nu}{2 \pi} \int\left[\tan ^{-1}\left(\frac{2 K(h) \xi^{*}}{K(h)^{2}-\xi^{* 2}}\right)+\pi\right]_{\Sigma_{h}} d \theta .
\end{gathered}
$$

It is shown that $n_{0}\left(E, m, p_{\theta}, \theta\right)$ represents the original 't Hooft particles [13] [32], and $n_{1}\left(E, m, p_{\theta}, \theta\right)$ the scattering particles [13].

For an ensemble of states of the scalar field, the free energy can be expressed as

$$
\begin{aligned}
\beta F & =\int d m \int d p_{\theta} \int d n\left(E, m, p_{\theta}\right) \ln \left[1-e^{-\beta\left(E-\Omega_{0} m\right)}\right] \\
& =\int d m \int d p_{\theta} \int d n\left(E+\Omega_{0} m, m, p_{\theta}\right) \ln \left(1-e^{-\beta E}\right) \\
& =-\beta \int d m \int d p_{\theta} \int \frac{n_{0}\left(E+\Omega_{0} m, m, p_{\theta}\right)+n_{1}\left(E+\Omega_{0} m, m, p_{\theta}\right)}{e^{\beta E}-1} d E \\
& =-\beta \int \frac{n_{0}(E)+n_{1}(E)}{e^{\beta E}-1} d E \\
& =\beta F_{0}+\beta F_{1}
\end{aligned}
$$

with 


$$
\begin{aligned}
n_{0}(E) & =\int d m \int d p_{\theta} \int n_{0}\left(E+\Omega_{0} m, m, p_{\theta}\right) \\
& =\frac{1}{3 \pi} \int d \theta \int_{r_{H}+h}^{r_{E}} \frac{\sqrt{g_{4}}\left[E^{2}+\left(g_{t t}-\frac{g_{t \varphi}^{2}}{g_{\varphi \varphi}}\right)\left(1+\frac{g_{\varphi \varphi^{2}\left(\Omega-\Omega_{0}\right)^{2}}}{g_{t t} g_{\varphi \varphi}-g_{t \varphi}^{2}}\right) M^{2}(r, \theta)\right]^{\frac{3}{2}}}{\left[\left(g_{t t}-\frac{g_{t \varphi}^{2}}{g_{\varphi \varphi}}\right)\left(1+\frac{g_{\varphi \varphi}{ }^{2}\left(\Omega-\Omega_{0}\right)^{2}}{g_{t t} g_{\varphi \varphi}-g_{t \varphi}^{2}}\right)\right]^{2}}, \\
n_{1}(E) & =\int d m \int d p_{\theta} \int n_{1}\left(E+\Omega_{0} m, m, p_{\theta}\right) \\
& \approx \frac{\nu}{\pi} \int d \theta\left\{\frac{\sqrt{g_{\theta \theta} g_{\varphi \varphi}}\left[\xi^{*} \bar{K}-\xi^{* 2} \tan ^{-1}\left(\frac{\bar{K}}{\xi^{*}}\right)+\frac{\bar{K}^{2}}{2} \tan ^{-1}\left(\frac{2 \xi^{*} \bar{K}}{K^{2}-\xi^{* 2}}\right)\right]}{\left(g_{t t}-\frac{g_{t \varphi}^{2}}{g_{\varphi \varphi}}\right)\left(1+\frac{g_{\varphi \varphi^{2}\left(\Omega-\Omega_{0}\right)^{2}}}{g_{t t} g_{\varphi \varphi}-g_{t \varphi}^{2}}\right)}\right\}_{\Sigma_{h}} .
\end{aligned}
$$

Taking the integration $r$ of Eq. (2.21) for $\Omega_{0}=\Omega_{H}$ and focusing only on the divergent contribution at horizon, we find

$$
\begin{aligned}
n_{0}(E)= & -\frac{1}{2 \pi} \int d \theta\left\{\sqrt{g_{\theta \theta} g_{\varphi \varphi}}\left[\frac{2}{3}\left(\frac{E \beta_{H}}{4 \pi}\right)^{3} C(r, \theta)+M^{2}(r, \theta)\left(\frac{E \beta_{H}}{4 \pi}\right)\right]\right. \\
& \left.\times \ln \left(\frac{E^{2}}{E_{m i n}^{2}}\right)\right\}_{r_{H}}-\frac{1}{3 \pi}\left(\frac{\beta_{H}}{4 \pi}\right) \int d \theta\left\{\sqrt{g_{\theta \theta} g_{\varphi \varphi}} M^{2}(r, \theta)\left(E-\frac{E^{3}}{E_{m i n}^{2}}\right)\right\}_{r_{H}}, \\
n_{1}(E)= & \frac{\nu}{\pi} \int d \theta\left\{\frac { ( \xi ^ { * } M ( r , \theta ) ) ^ { 2 } \sqrt { g _ { \theta \theta } g _ { \varphi \varphi } } } { E _ { m i n } ^ { 2 } } \left[\tan ^{-1}\left(\frac{\bar{K}}{\xi^{*}}\right)-\frac{\bar{K}}{\xi^{*}}\right.\right. \\
& \left.\left.-\frac{\bar{K}^{2}}{2 \xi^{* 2}} \tan ^{-1}\left(\frac{2 \xi^{*} \bar{K}}{\bar{K}^{2}-\xi^{* 2}}\right)\right]\right\}_{r_{H}}
\end{aligned}
$$

where

$$
\begin{aligned}
C(r, \theta)= & \frac{\partial^{2} g^{r r}}{\partial r^{2}}+\frac{3}{2} \frac{\partial g^{r r}}{\partial r} \frac{\partial \ln f}{\partial r}-\frac{1}{2} \frac{\partial g^{r r}}{\partial r}\left(\frac{1}{g_{\theta \theta}} \frac{\partial g_{\theta \theta}}{\partial r}\right. \\
& \left.+\frac{1}{g_{\varphi \varphi}} \frac{\partial g_{\varphi \varphi}}{\partial r}\right)-\frac{2 g_{\varphi \varphi}}{f}\left[\frac{\partial}{\partial r}\left(\frac{g_{t \varphi}}{g_{\varphi \varphi}}\right)\right]^{2}, \\
E_{\text {min }}^{2}= & -M^{2}\left(r_{H}, \theta\right)\left(g_{t t}-\frac{g_{t \varphi^{2}}}{g_{\varphi \varphi}}\right)_{\Sigma_{h}}, \\
\bar{K}^{2}= & E^{2}+\left(g_{t t}-\frac{g_{t \varphi}^{2}}{g_{\varphi \varphi}}\right)_{\Sigma_{h}} M^{2}\left(r_{H}, \theta\right) .
\end{aligned}
$$

Let use the Pauli-Villars regularization scheme [14] by introducing five regulator fields $\left\{\phi_{i}, i=1, \ldots, 5\right\}$ of different statistics with masses $\left\{\mu_{i}, i=1, \ldots, 5\right\}$ dependent on the UV 
cutoff [14] and with the same nonminimal coupling $\left\{\xi_{i}=\xi, i=0, \ldots, 5\right\}$. If we rewrite the original scalar field $\phi=\phi_{0}$ and $\mu=\mu_{0}$, then these fields satisfy $\Sigma_{i=0}^{5} \triangle_{i}=0$ and $\Sigma_{i=0}^{5} \triangle_{i} \mu_{i}^{2}=$ 0 , where $\triangle_{0}=\triangle_{3}=\triangle_{4}=+1$ for the commuting fields and $\triangle_{1}=\triangle_{2}=\triangle_{5}=-1$ for the anticommuting fields. Since each of the fields makes a contribution to the free energy of Eq. (2.20), and the total free energy becomes

$$
\beta \bar{F}=\sum_{i=0}^{5} \triangle_{i} \beta F_{i}=\beta \bar{F}_{0}+\beta \bar{F}_{1} .
$$

Substituting Eqs. (2.20), (2.23), and (2.24) into (2.27) and integrating over E we find

$$
\begin{aligned}
\bar{F}_{0}= & -\frac{1}{48} \frac{\beta_{H}}{\beta^{2}} \int d \theta\left\{\sqrt{g_{\theta \theta} g_{\varphi \varphi}}\right\}_{r_{H}} \sum_{i=0}^{5} \triangle_{i} M_{i}^{2}\left(r_{H}, \theta\right) \ln M_{i}^{2}\left(r_{H}, \theta\right) \\
& -\frac{1}{2880} \frac{\beta_{H}^{3}}{\beta^{4}} \int d \theta\left\{\sqrt { g _ { \theta \theta } g _ { \varphi \varphi } } \left[\frac{\partial^{2} g^{r r}}{\partial r^{2}}+\frac{3}{2} \frac{\partial g^{r r}}{\partial r} \frac{\partial \ln f}{\partial r}-\frac{1}{2} \frac{\partial g^{r r}}{\partial r}\left(\frac{1}{g_{\theta \theta}} \frac{\partial g_{\theta \theta}}{\partial r}\right.\right.\right. \\
& \left.\left.\left.+\frac{1}{g_{\varphi \varphi}} \frac{\partial g_{\varphi \varphi}}{\partial r}\right)-\frac{2 g_{\varphi \varphi}}{f}\left[\frac{\partial}{\partial r}\left(\frac{g_{t \varphi}}{g_{\varphi \varphi}}\right)\right]^{2}\right]\right\} \sum_{r_{H}}^{5} \triangle_{i} \ln M_{i}^{2}\left(r_{H}, \theta\right) \\
\bar{F}_{1} & =-\frac{|\xi|}{4 \beta} \int d \theta\left\{\sqrt{g_{\theta \theta} g_{\varphi \varphi}}\right\}_{r_{H}} \sum_{i=0}^{5} \triangle_{i} M_{i}^{2}\left(r_{H}, \theta\right) \ln M_{i}^{2}\left(r_{H}, \theta\right),
\end{aligned}
$$

where $M_{i}^{2}\left(r_{H}, \theta\right)=\mu_{i}^{2}-\left(\frac{1}{6}-\xi\right) R$. The total entropy at the Hawking temperature $\frac{1}{\beta}=\frac{1}{\beta_{H}}$ is given by

$$
\begin{aligned}
S^{S M}= & {\left[\beta^{2} \frac{\partial \bar{F}}{\partial \beta}\right]_{\beta=\beta_{H}}=\left[\beta^{2} \frac{\partial\left(\bar{F}_{0}+\bar{F}_{1}\right)}{\partial \beta}\right]_{\beta=\beta_{H}} } \\
= & \frac{1}{4}\left(\frac{1}{6}+|\xi|\right) \int d \theta\left\{\sqrt{g_{\theta \theta} g_{\varphi \varphi}}\right\}_{r_{H}} \sum_{i=0}^{5} \triangle_{i} M_{i}^{2}\left(r_{H}, \theta\right) \ln M_{i}^{2}\left(r_{H}, \theta\right) \\
& +\frac{1}{720} \int d \theta\left\{\sqrt { g _ { \theta \theta } g _ { \varphi \varphi } } \left[\frac{\partial^{2} g^{r r}}{\partial r^{2}}+\frac{3}{2} \frac{\partial g^{r r}}{\partial r} \frac{\partial \ln f}{\partial r}-\frac{1}{2} \frac{\partial g^{r r}}{\partial r}\left(\frac{1}{g_{\theta \theta}} \frac{\partial g_{\theta \theta}}{\partial r}\right.\right.\right. \\
& \left.\left.\left.+\frac{1}{g_{\varphi \varphi}} \frac{\partial g_{\varphi \varphi}}{\partial r}\right)-\frac{2 g_{\varphi \varphi}}{f}\left[\frac{\partial}{\partial r}\left(\frac{g_{t \varphi}}{g_{\varphi \varphi}}\right)\right]^{2}\right]\right\}_{r_{H}} \sum_{i=0}^{5} \triangle_{i} \ln M_{i}^{2}\left(r_{H}, \theta\right) .
\end{aligned}
$$

Using the assumption that the scalar curvature at the horizon is much smaller than each $\mu_{i}$ and noting that the area of the event horizon can be expressed as $A_{\Sigma}=$ $\int d \varphi \int d \theta\left\{\sqrt{g_{\theta \theta} g_{\varphi \varphi}}\right\}_{r_{H}}$, we obtain following expression

$$
\begin{aligned}
& S^{S M}= \\
& \frac{A_{\Sigma}}{48 \pi}(1+6|\xi|) \sum_{i=0}^{5} \triangle_{i} \mu_{i}^{2} \ln \mu_{i}^{2}+\left\{-\frac{1}{8 \pi}\left(\frac{1}{6}+|\xi|\right)\left(\frac{1}{6}-\xi\right) \int d \varphi d \theta\left(R \sqrt{g_{\theta \theta} g_{\varphi \varphi}}\right)_{r_{H}}\right.
\end{aligned}
$$




$$
\begin{aligned}
& +\int d \varphi d \theta\left[\frac { \sqrt { g _ { \theta \theta } g _ { \varphi \varphi } } } { 1 4 4 0 \pi } \left(\frac{\partial^{2} g^{r r}}{\partial r^{2}}+\frac{3}{2} \frac{\partial g^{r r}}{\partial r} \frac{\partial \ln f}{\partial r}-\frac{1}{2} \frac{\partial g^{r r}}{\partial r}\left(\frac{1}{g_{\theta \theta}} \frac{\partial g_{\theta \theta}}{\partial r}+\frac{1}{g_{\varphi \varphi}} \frac{\partial g_{\varphi \varphi}}{\partial r}\right)\right.\right. \\
& \left.\left.\left.-\frac{2 g_{\varphi \varphi}}{f}\left[\frac{\partial}{\partial r}\left(\frac{g_{t \varphi}}{g_{\varphi \varphi}}\right)\right]^{2}\right)\right]_{r_{H}}\right\} \sum_{i=0}^{5} \triangle_{i} \ln \mu_{i}^{2} .
\end{aligned}
$$

If we set $\xi=0$ we known that Eq. (2.31) gives result of the Ref. 32.

The main aim of this paper is to seek the relation between the statistical-mechanical and the thermodynamical entropies. In order to do that, we will cast result (2.31) into another form. In appendix $\mathrm{A}$ we proved

$$
\begin{aligned}
& \left\{\frac{\partial^{2} g^{r r}}{\partial r^{2}}+\frac{3}{2} \frac{\partial g^{r r}}{\partial r} \frac{\partial \ln f}{\partial r}-\frac{1}{2} \frac{\partial g^{r r}}{\partial r}\left(\frac{1}{g_{\theta \theta}} \frac{\partial g_{\theta \theta}}{\partial r}+\frac{1}{g_{\varphi \varphi}} \frac{\partial g_{\varphi \varphi}}{\partial r}\right)-\frac{2 g_{\varphi \varphi}}{f}\left[\frac{\partial}{\partial r}\left(\frac{g_{t \varphi}}{g_{\varphi \varphi}}\right)\right]^{2}\right\}_{r_{H}} \\
& =R_{a a}\left(r_{H}, \theta\right)-2 R_{a b a b}\left(r_{H}, \theta\right),
\end{aligned}
$$

where $R_{a a}=\sum_{a=1}^{2} R_{\mu \nu} n_{a}^{\mu} n_{a}^{\nu}, \quad R_{a b a b}=\sum_{a, b=1}^{2} R_{\mu \nu \lambda \rho} n_{a}^{\mu} n_{b}^{\nu} n_{a}^{\lambda} n_{b}^{\rho}$ are the projections of the curvature onto the subspace normal to the horizon surface and $\left\{n_{a}^{\mu}, a=1,2\right\}$ (see Eq. (A1) in Appendix A) are a pair of vectors orthogonal to the event horizon $\Sigma$. From (2.32) we know that Eq. (2.31) can be rewritten as

$$
\begin{aligned}
S^{S M} & =\frac{A_{\Sigma}}{48 \pi}(1+6|\xi|) \sum_{i=0}^{5} \triangle_{i} \mu_{i}^{2} \ln \mu_{i}^{2}+\left\{-\frac{1}{8 \pi}\left(\frac{1}{6}+|\xi|\right)\left(\frac{1}{6}-\xi\right) \int_{\Sigma} R\right. \\
& \left.+\int_{\Sigma}\left[\frac{1}{1440 \pi}\left(\sum_{a=1}^{2} R_{\mu \nu} n_{a}^{\mu} n_{a}^{\nu}-2 \sum_{a, b=1}^{2} R_{\mu \nu \lambda \rho} n_{a}^{\mu} n_{b}^{\nu} n_{a}^{\lambda} n_{b}^{\rho}\right)\right]\right\} \sum_{i=0}^{5} \triangle_{i} \ln \mu_{i}^{2} .
\end{aligned}
$$

This is the expression of the statistical-mechanical entropy arising from the nonminimally coupled scalar fields in the four-dimensional nonextreme stationary axisymmetric black hole. It is of interest to note that Eq. (2.33) possesses same form as the corresponding result of the static black hole [13].

\section{THERMODYNAMICAL ENTROPY OF THE NONEXTREME STATIONARY AXISYMMETRIC BLACK HOLES}

Mann and Solodukhin [11] showed that an Euclidean manifold which is obtained by Wick rotation of the Kerr-Newman geometry has a conical singularity. By using the conical singularities method they obtained the tree-level thermodynamical entropy $S^{T D}\left(G_{B}, c_{B}^{i}\right)$ and its one-loop quantum corrections $S_{\text {div }}^{T D}$ for the Kerr-Newman black hole due to minimally coupled scalar field. Which are respectively given by

$$
\begin{aligned}
S^{T D}\left(G_{B}, c_{B}^{i}\right)= & \frac{A_{\Sigma}}{4 G_{B}}-8 \pi \int_{\Sigma}\left[\left(c_{B}^{1} R+\frac{c_{B}^{2}}{2} \sum_{a=1}^{2} R_{\mu \nu} n_{i}^{\mu} n_{i}^{\nu}\right.\right. \\
& \left.\left.+c_{B}^{3} \sum_{a, b=1}^{2} R_{\mu \nu \alpha \beta} n_{i}^{\mu} n_{j}^{\nu} n_{i}^{\alpha} n_{j}^{\beta}\right)\right]
\end{aligned}
$$




$$
\begin{aligned}
S_{\text {div }}^{T D}= & \frac{A_{\Sigma}}{48 \pi \epsilon^{2}}+\left\{\frac{1}{144 \pi} \int_{\Sigma} R-\frac{1}{45} \frac{1}{16 \pi} \int_{\Sigma}\left(\sum_{a=1}^{2} R_{\mu \nu} n_{i}^{\mu} n_{i}^{\nu}-2 \sum_{a, b=1}^{2} R_{\mu \nu \alpha \beta} n_{i}^{\mu} n_{j}^{\nu} n_{i}^{\alpha} n_{j}^{\beta}\right)\right. \\
& \left.-\frac{1}{90} \frac{1}{16 \pi} \int_{\Sigma}\left(K^{a} K^{a}\right)+\frac{1}{24 \pi}\left(\lambda_{1}-\frac{\lambda_{2}}{30}\right) \int_{\Sigma}\left(K^{a} K^{a}-2 \operatorname{tr}(K . K)\right)\right\} \ln \frac{L}{\epsilon},
\end{aligned}
$$

where $G_{B}, c_{B}^{i},(i=1,2,3)$ represent bare constants (tree-level), $K_{\mu \nu}^{a}=-\gamma_{\mu}^{\alpha} \gamma_{\nu}^{\beta} \nabla_{\alpha} n_{\beta}^{a}$ is the extrinsic curvature, and $K^{a}=g^{\mu \nu} K_{\mu \nu}^{a}$ is the trace of the extrinsic curvature [11]. We find that all terms which relate to the extrinsic curvature in result (3.2) are equal to zero if one insert the Kerr-Newman metric [11] (or the Einstein-Maxwell dilaton-axion metric [27]) into them.

Now we proceed to extend the one-loop quantum correction (3.2) to nonminimally coupled scalar fields for the general nonextreme stationary axisymmetric black hole.

In order to cast the metric (2.1) into Mann-Solodukhin's form [11, we define a pair of vectors as follows

$$
K=(1, \quad 0, \quad 0, \quad \tilde{\Omega}), \quad \tilde{K}=(F, \quad 0, \quad 0, \quad 1)
$$

with

$$
\begin{gathered}
F=-\frac{g_{t \varphi}+\tilde{\Omega} g_{\varphi \varphi}}{g_{t t}+\tilde{\Omega} g_{t \varphi}} \\
\tilde{\Omega}=\frac{g_{t t} \sqrt{g_{t \varphi}^{2}-g_{t t} g_{\varphi \varphi}+g_{t t} g_{\theta \theta} \sin ^{2} \theta}}{g_{t t} g_{\varphi \varphi}-g_{t \varphi}^{2}-g_{t \varphi} \sqrt{g_{t \varphi}^{2}-g_{t t} g_{\varphi \varphi}+g_{t t} g_{\theta \theta} \sin ^{2} \theta}} .
\end{gathered}
$$

we find in the region $r_{H} \leq r<\infty$ that $F=a \sin ^{2} \theta$ and $\tilde{\Omega}=\frac{a}{r^{2}+a^{2}}$ for the Kerr and the Kerr-Newman black holes, and $F=\operatorname{asin}^{2} \theta$ and $\tilde{\Omega}=\frac{a}{r^{2}-2 d r+a^{2}}$ for the Einstein-Maxwell dilaton-axion black hole. In general, on the event horizon, $\tilde{\Omega}\left(r_{H}\right)=\Omega_{H}=-\left(\frac{g_{t \varphi}}{g_{\varphi \varphi}}\right)_{r_{H}}$, where $\Omega_{H}$ is the angular velocity of the horizon. We can prove that $K$ and $\tilde{K}$ are a pair of the Killing vectors on the horizon. What we need in following is just the properties of the vectors $K$ and $\tilde{K}$ near the event horizon.

The one forms dual to $K$ and $\tilde{K}$ are respectively given by

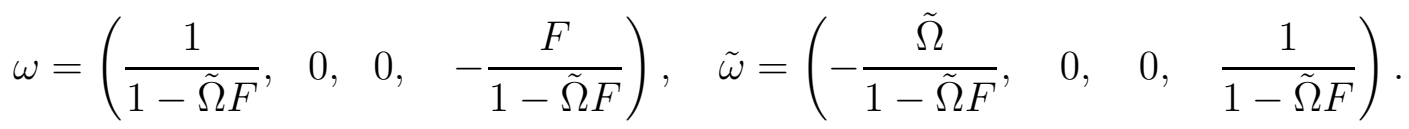

Thus, the metric (2.1) can be written as

$$
d s^{2}=\left(g_{t t}+2 \tilde{\Omega} g_{t \varphi}+\tilde{\Omega}^{2} g_{\varphi \varphi}\right) \omega^{2}+g_{r r} d r^{2}+g_{\theta \theta}\left(d \theta^{2}+\sin ^{2} \theta \tilde{\omega}^{2}\right) .
$$

Euclideanize the metric by setting $t=i \tau, \tilde{\Omega}=i \hat{\Omega}$, and $F=i \hat{F}$, then the Euclidean vectors (3.3) and the corresponding one-forms (3.6) take the form

$$
K=(1, \quad 0, \quad 0,-\hat{\Omega}), \quad \tilde{K}=(\hat{F}, \quad 0, \quad 0, \quad 1)
$$




$$
\omega=\left(\frac{1}{1+\hat{\Omega} \hat{F}}, \quad 0, \quad 0, \quad-\frac{\hat{F}}{1+\hat{\Omega} \hat{F}}\right), \quad \tilde{\omega}=\left(\begin{array}{llll}
\frac{\hat{\Omega}}{1+\hat{\Omega} \hat{F}} & 0, & 0, & \frac{1}{1+\hat{\Omega} \hat{F}}
\end{array}\right) .
$$

The metric (3.7) becomes

$$
\begin{aligned}
d s^{2} & =\hat{H}(r, \theta)\left(r-\hat{r}_{H}\right)(d \tau-\hat{F} d \varphi)^{2}+\frac{1}{\hat{G}_{2}(r, \theta)\left(r-\hat{r}_{H}\right)} d r^{2} \\
& +\hat{g}_{\theta \theta}\left(d \theta^{2}+\sin ^{2} \theta \tilde{\omega}^{2}\right),
\end{aligned}
$$

with

$$
\hat{H}(r, \theta)\left(r-\hat{r}_{H}\right) \equiv \frac{\hat{g}_{t t}+2 \hat{\Omega} \hat{g}_{t \varphi}+\hat{\Omega}^{2} \hat{g}_{\varphi \varphi}}{(1+\hat{\Omega} \hat{F})^{2}} .
$$

It is useful to introduce a new variable

$$
\left(r-\hat{r}_{H}\right)=\frac{x^{2}}{4}
$$

up to term $o\left(x^{2}\right)$ the metric (3.10) can be expressed as

$$
d s^{2}=\frac{1}{\hat{G}_{2}\left(\hat{r}_{H}, \theta\right)} d s_{c 2}^{2}+d s_{\Sigma}^{2}
$$

with

$$
\begin{aligned}
d s_{c 2}^{2} & =d x^{2}+\frac{\hat{H}\left(\hat{r}_{H}, \theta\right) \hat{G}_{2}\left(\hat{r}_{H}, \theta\right) x^{2}}{4}\left(d \tau-\hat{F}\left(\hat{r}_{H}, \theta\right) d \varphi\right)^{2}, \\
d s_{\Sigma}^{2} & =\hat{g}_{\theta \theta}\left(d \theta^{2}+\sin ^{2} \theta \tilde{\omega}^{2}\right),
\end{aligned}
$$

Introducing new angle coordinate $d \chi=\frac{\beta_{H}}{\beta} \sqrt{\frac{4}{\hat{H} \hat{G}_{2}}}(d \tau-\hat{F} d \varphi)$, Eq. (3.12) reads

$$
d s_{c 2 \alpha}^{2}=d x^{2}+\alpha^{2} x^{2} d \chi^{2},
$$

From Euclidean metric (3.10) we can define a pair of vectors orthogonal to the horizon

$$
\begin{aligned}
& n_{1}^{\mu}=\left(\begin{array}{lll}
0, & \sqrt{\hat{g}^{r r}}, \quad 0, \quad 0
\end{array}\right) \\
& n_{2}^{\mu}=\left(\frac{1}{\sqrt{\hat{g}_{t t}+2 \tilde{\Omega} \hat{g}_{t \varphi}+\hat{\Omega}^{2} \hat{g}_{\varphi \varphi}}}, \quad 0, \quad 0, \quad \frac{\hat{\Omega}}{\sqrt{\hat{g}_{t t}+2 \hat{\Omega} \hat{g}_{t \varphi}+\hat{\Omega}^{2} \hat{g}_{\varphi \varphi}}}\right), \\
& n_{\mu}^{1}=\left(\begin{array}{lll}
0, & \frac{1}{\sqrt{\hat{g}^{r r}}}, \quad 0, \quad 0
\end{array}\right) \text {, } \\
& n_{\mu}^{2}=\left(\frac{\sqrt{\hat{g}_{t t}+2 \hat{\Omega} \hat{g}_{t \varphi}+\hat{\Omega}^{2} \hat{g}_{\varphi \varphi}}}{1+\hat{\Omega} \hat{F}}, \quad 0, \quad 0, \quad-\frac{\hat{F} \sqrt{\hat{g}_{t t}+2 \hat{\Omega} \hat{g}_{t \varphi}+\hat{\Omega}^{2} \hat{g}_{\varphi \varphi}}}{1+\hat{\Omega} \hat{F}}\right) .
\end{aligned}
$$

It is helpful to note that Eqs. (3.13), (3.11), (3.14), (3.15) and (3.16) take the similar form as Eqs. (2.8), (2.9), (3.1), (A1,A2) and (A3, A4) in Ref. [11], respectively. Therefore, 
the discussions for the general nonextreme stationary black hole (2.1) is parallel with that for the Kerr-Newman black hole 11.

Following Solodukhin's [6] and Mann and Solodukhin's [11] discussions, and employing the results obtained in Appendix B

$$
\begin{aligned}
\left(K^{a} K^{a}\right)_{r_{H}} & =0, \\
{[\operatorname{tr}(K . K)]_{r_{H}} } & =\left(K_{\mu \nu}^{a} K_{a}^{\mu \nu}\right)_{r_{H}}=0,
\end{aligned}
$$

we obtain one-loop quantum correction to the thermodynamical entropy arising from the nonminimally coupled scalar field for the general nonextreme stationary axisymmetric black hole as follows

$$
\begin{aligned}
S_{\text {div }}^{T D} & =\frac{A_{\Sigma}}{8 \pi \epsilon^{2}}\left(\frac{1}{6}-\xi\right)+\left\{\frac{1}{4 \pi}\left(\frac{1}{6}-\xi\right)^{2} \int_{\Sigma} R\right. \\
& \left.-\frac{1}{45} \frac{1}{16 \pi} \int_{\Sigma}\left(\sum_{a=1}^{2} R_{\mu \nu} n_{a}^{\mu} n_{a}^{\nu}-2 \sum_{a, b=1}^{2} R_{\mu \nu \lambda \rho} n_{a}^{\mu} n_{b}^{\nu} n_{a}^{\lambda} n_{b}^{\rho}\right)\right\} \ln \frac{L}{\epsilon} .
\end{aligned}
$$

The expressions (3.17) and (3.18) has been backed to real values of the parameters $t, \tilde{\Omega}$ and $F$. It is of interest to note that for the general nonextreme stationary axisymmetric black hole the contributions of the quadratic combinations of the extrinsic curvature of the horizon to the entropy, as for the Kerr-Newman black hole [11], are zero when we define a pair of vectors orthogonal to the horizon $\Sigma$ and dual to one-forms. Therefore, the logarithmically divergent part of the result (3.18) depends only on projections of the curvature onto subspace normal to the horizon as the static black hole does.

\section{SUMMARY AND DISCUSSION}

Since the Pauli-Villars regularization scheme causes a factor $-\frac{1}{2}$ in second part in the Eq. (2.33), we know from above discussions that the statistical-mechanical entropy (2.33) coincides with the one-loop quantum correction to thermodynamical entropy (3.18) for $\xi \leq 0$ coupling.

We now seek for relation between the statistical-mechanical entropy of quantum excitations of the stationary axisymmetric black hole and its thermodynamical entropy. We first renormalize the thermodynamical entropy by using standard approach [34] [6] [14] [1]. Combing the tree-level entropy (3.1) with one-loop correction (3.18) one find that the divergence can be absorbed in the renormalization of the coupling constants

$$
\begin{aligned}
\frac{1}{G_{\text {ren }}} & =\frac{1}{G_{B}}+\frac{1}{2 \pi \epsilon^{2}}\left(\frac{1}{6}-\xi\right), \\
c_{\text {ren }}^{1} & =c_{B}^{1}-\frac{1}{32 \pi^{2}}\left(\frac{1}{6}-\xi\right)^{2} \ln \frac{L}{\epsilon}, \\
c_{\text {ren }}^{2} & =c_{B}^{2}+\frac{1}{32 \pi^{2}} \frac{1}{90} \ln \frac{L}{\epsilon}, \\
c_{\text {ren }}^{3} & =c_{B}^{3}-\frac{1}{32 \pi^{2}} \frac{1}{90} \ln \frac{L}{\epsilon} .
\end{aligned}
$$


From Eqs. (3.1), (3.18) and (4.1) we have

$$
\begin{aligned}
& S^{T D}\left(G_{r e n}, c_{r e n}^{i}\right)=S^{T D}\left(G_{B}, c_{B}^{i}\right)+S_{d i v}^{T D} \\
= & \frac{A_{\Sigma}}{4 G_{r e n}}-8 \pi \int_{\Sigma}\left(c_{r e n}^{1} R+\frac{c_{r e n}^{2}}{2} \sum_{a=1}^{2} R_{\mu \nu} n_{i}^{\mu} n_{i}^{\nu}+c_{r e n}^{3} \sum_{a, b=1}^{2} R_{\mu \nu \alpha \beta} n_{i}^{\mu} n_{j}^{\nu} n_{i}^{\alpha} n_{j}^{\beta}\right) .
\end{aligned}
$$

Since we considered the case that terms quadratic in curvature are preserved in the renormalized action, the black hole entropy can be expressed as(see Refs. [35]- [38])

$$
\begin{aligned}
S^{B H}\left(G_{r e n}, c_{r e n}^{i}\right) & =S^{T D}\left(G_{r e n}, c_{r e n}^{i}\right), \\
S^{B H}\left(G_{B}, c_{B}^{i}\right) & =S^{T D}\left(G_{B}, c_{B}^{i}\right),
\end{aligned}
$$

and the Bekenstein-Hawking entropy is

$$
S^{B H}=\frac{A_{\Sigma}}{4 G_{r e n}} .
$$

Noting $S_{\text {div }}^{T D}=S^{S M}$ for $\xi \leq 0$, we obtain for $\xi \leq 0$ the relation

$$
S^{B H}\left(G_{r e n}, c_{r e n}^{i}\right)=S^{B H}\left(G_{B}, c_{B}^{i}\right)+S^{S M},
$$

which agrees the static black hole results shown in Refs. [14] [10] [39] [5] [40]. It is shown that the presence of the bare pure geometrical contribution $S^{B H}\left(G_{B}, c_{B}^{i}\right)$ evidently excludes the possibility to identify $S^{B H}\left(G_{r e n}, c_{r e n}^{i}\right)$ with $S^{S M}$.

In conclusion, on the one hand by means of the BWM in which the original Dirichlet condition was replaced by a scattering ansatz for the field functions at the event horizon and with the Pauli-Villars regularization scheme, the statistical-mechanical entropy arising from the nonminimally coupled scalar fields which rotate with the angular velocity $\Omega_{0}=\Omega_{H}$ in the general four-dimensional nonextreme stationary axisymmetric black hole space-time is studied. The result can be expressed as either Eq. (2.31) or Eq. (2.33).

On the other hand by using the conical singularities method we extend Mann and Solodukhin's result for the Kerr-Newman black hole [11] to the general nonextreme stationary axisymmetric black hole and the nonminimally coupled scalar field. Nevertheless, we find that the logarithmically part in Eq. (3.18) depends only on projections of the curvature onto subspace normal to the horizon since the contributions of the quadratic combinations of the extrinsic curvature of the horizon are zero when we define a pair of vectors orthogonal to the event horizon $\Sigma$ and dual to one-forms.

By comparing the statistical-mechanical entropy (2.33) and the thermodynamical entropy (3.18) we show that, for the general nonextreme stationary axisymmetric black hole, the statistical mechanical entropy and the one-loop correction to the thermodynamic entropy are equivalent for the coupling $\xi \leq 0$. It is an interesting result that the entropies possesses the same form as static black hole does [6] if we express the entropy with the projections of the curvature onto subspace normal to the horizon.

Combing the tree-level entropy (3.1) with one-loop correction (3.18), the divergence can be absorbed in the renormalization of the gravitational and coupling constants. After renormalized with standard scheme, a relation between the statistical-mechanical entropy of quantum excitations of the nonextreme stationary axisymmetric black hole and its thermodynamical entropy for the case $\xi \leq 0$ is obtained. The relation agrees the results for the static black hole and fills in the gaps mentioned in Ref. [1]. 


\section{APPENDIX A: PROJECTIONS OF THE CURVATURE ONTO THE SUBSPACE NORMAL TO THE HORIZON SURFACE}

From vectors (3.3), dual vectors (3.6) and metric (3.7), we can define a pair of orthonormal vectors

$$
\begin{aligned}
& n_{1}^{\mu}=\left(\begin{array}{llll}
0, & \sqrt{g^{r r}}, \quad 0, & 0
\end{array}\right), \\
& n_{2}^{\mu}=\left(\frac{1}{\sqrt{-\left(g_{t t}+2 \tilde{\Omega} g_{t \varphi}+\tilde{\Omega}^{2} g_{\varphi \varphi}\right)}}, \quad 0, \quad 0, \quad \frac{\tilde{\Omega}}{\sqrt{-\left(g_{t t}+2 \tilde{\Omega} g_{t \varphi}+\tilde{\Omega}^{2} g_{\varphi \varphi}\right)}}\right), \\
& n_{\mu}^{1}=\left(\begin{array}{lll}
0, \quad \frac{1}{\sqrt{g^{r r}}}, \quad 0, & 0
\end{array}\right), \\
& n_{\mu}^{2}=\left(\frac{\sqrt{-\left(g_{t t}+2 \tilde{\Omega} g_{t \varphi}+\tilde{\Omega}^{2} g_{\varphi \varphi}\right)}}{1-\tilde{\Omega} F}, 0, \quad 0, \quad-\frac{F \sqrt{-\left(g_{t t}+2 \tilde{\Omega} g_{t \varphi}+\tilde{\Omega}^{2} g_{\varphi \varphi}\right)}}{1-\tilde{\Omega} F}\right) .
\end{aligned}
$$

After tediously calculation, the projections of the curvature onto the subspace normal to the horizon surface

$$
R_{a a}=\sum_{a=1}^{2} R_{\mu \nu} n_{a}^{\mu} n_{a}^{\nu}, \quad R_{a b a b}=\sum_{a=1}^{2} R_{\mu \nu \lambda \rho} n_{a}^{\mu} n_{b}^{\nu} n_{a}^{\lambda} n_{b}^{\rho},
$$

can be expressed by using the metric as

$$
\begin{aligned}
R_{a a}\left(r_{H}, \theta\right)= & {\left[\frac{g_{t \varphi}^{2}}{f g_{\varphi \varphi}^{3}}\left(\frac{\partial g_{\varphi \varphi}}{\partial r}\right)^{2}-\frac{2 g_{t \varphi}}{f g_{\varphi \varphi}^{2}} \frac{\partial g_{\varphi \varphi}}{\partial r} \frac{\partial g_{t \varphi}}{\partial r}+\frac{1}{f g_{\varphi \varphi}}\left(\frac{\partial g_{t \varphi}}{\partial r}\right)^{2}\right.} \\
& \left.-\frac{3}{2} \frac{\partial \ln f}{\partial r} \frac{\partial g^{r r}}{\partial r}-\frac{1}{2 g_{\theta \theta}} \frac{\partial g_{\theta \theta}}{\partial r} \frac{\partial g^{r r}}{\partial r}-\frac{1}{2 g_{\varphi \varphi}} \frac{\partial g_{\varphi \varphi}}{\partial r} \frac{\partial g^{r r}}{\partial r}-\frac{\partial^{2} g^{r r}}{\partial r^{2}}\right]_{r_{H}} \\
R_{a b a b}\left(r_{H}, \theta\right)= & \frac{1}{f}\left[\frac{3 g_{t \varphi}^{2}}{2 g_{\varphi \varphi}^{3}}\left(\frac{\partial g_{\varphi \varphi}}{\partial r}\right)^{2}-\frac{3 g_{t \varphi}}{g_{\varphi \varphi}^{2}} \frac{\partial g_{\varphi \varphi}}{\partial r} \frac{\partial g_{t \varphi}}{\partial r}\right. \\
& \left.+\frac{3}{2 g_{\varphi \varphi}}\left(\frac{\partial g_{t \varphi}}{\partial r}\right)^{2}-\frac{3}{2} \frac{\partial f}{\partial r} \frac{\partial g^{r r}}{\partial r}-f \frac{\partial^{2} g^{r r}}{\partial r^{2}}\right]_{r_{H}}
\end{aligned}
$$

Eqs. (A4) and (A5) yield

$$
\begin{aligned}
& R_{n n}\left(r_{H}, \theta\right)-2 R_{m n m n}\left(r_{H}, \theta\right)= \\
& \left\{\frac{\partial^{2} g^{r r}}{\partial r^{2}}+\frac{3}{2} \frac{\partial g^{r r}}{\partial r} \frac{\partial \ln f}{\partial r}-\frac{1}{2} \frac{\partial g^{r r}}{\partial r}\left(\frac{1}{g_{\theta \theta}} \frac{\partial g_{\theta \theta}}{\partial r}+\frac{1}{g_{\varphi \varphi}} \frac{\partial g_{\varphi \varphi}}{\partial r}\right)-\frac{2 g_{\varphi \varphi}}{f}\left[\frac{\partial}{\partial r}\left(\frac{g_{t \varphi}}{g_{\varphi \varphi}}\right)\right]^{2}\right\}_{r_{H}}
\end{aligned}
$$




\section{APPENDIX B: EXTRINSIC GEOMETRY OF THE HORIZON}

Using the vectors $n^{1}$ and $n^{2}$ defined by Eqs. (A1) and (A2) we can introduce induced metric

$$
\gamma_{\mu \nu}=g_{\mu \nu}-n_{\mu}^{1} n_{\nu}^{1}-n_{\mu}^{2} n_{\nu}^{2}
$$

The nonzero components of the induced metric are

$$
\begin{aligned}
\gamma_{t t} & =\frac{g_{t \varphi}^{2}}{g_{\varphi \varphi}}-f g^{r r}+\frac{f H g^{r r}}{\left(1+\frac{\tilde{\Omega}\left(g_{t \varphi}+g_{\varphi \varphi} \tilde{\Omega}\right)}{g_{t \varphi}^{2} / g_{\varphi \varphi}-f g^{r r}+g_{t \varphi} \tilde{\Omega}}\right)^{2}} \\
\gamma_{t \varphi} & =g_{t \varphi}+\frac{\left(f g_{\varphi \varphi} H g^{r r}\left(g_{t \varphi}+g_{\varphi \varphi} \tilde{\Omega}\right)\left(-g_{t \varphi}^{2}+f g_{\varphi \varphi} g^{r r}-g_{\varphi \varphi} g_{t \varphi} \tilde{\Omega}\right)\right)}{\left(-g_{t \varphi}^{2}+f g_{\varphi \varphi} g^{r r}-2 g_{\varphi \varphi} g_{t \varphi} \tilde{\Omega}-g_{\varphi \varphi}^{2} \tilde{\Omega}^{2}\right)^{2}} \\
\gamma_{\theta \theta} & =g_{\theta \theta}, \\
\gamma_{\varphi \varphi} & =g_{\varphi \varphi}+\frac{\left(f g_{\varphi \varphi}^{2} H g^{r r}\left(g_{t \varphi}+g_{\varphi \varphi} \tilde{\Omega}\right)^{2}\right.}{\left(-g_{t \varphi}^{2}+f g_{\varphi \varphi} g^{r r}-2 g_{\varphi \varphi} g_{t \varphi} \tilde{\Omega}-g_{\varphi \varphi}^{2} \tilde{\Omega}^{2}\right)^{2}}
\end{aligned}
$$

where $H=\left(1+\frac{g_{\varphi \varphi}(\Omega-\tilde{\Omega})^{2}}{g_{t t} g_{\varphi \varphi}-g_{t \varphi}^{2}}\right)$, and $\Omega=-\frac{g_{t \varphi}}{g_{\varphi \varphi}}$. With respect to the normal vectors $n_{\mu}^{a}, \quad(a=$ $1,2)$ we define the extrinsic curvature $k_{\mu \nu}^{a}=-\gamma_{\mu}^{\alpha} \gamma_{\nu}^{\beta} \nabla_{\alpha} n_{\beta}^{a}$. The nonzero components of the extrinsic curvature can be expressed as

$$
\begin{aligned}
& K_{t t}^{1}=-\frac{\tilde{\Omega}^{2}\left(g_{t \varphi}^{2}-f g_{\varphi \varphi} g^{r r}+g_{\varphi \varphi} g_{t \varphi} \tilde{\Omega}\right)^{2}}{2 f^{2} g_{\varphi \varphi}^{2} H^{2}\left(g^{r r}\right)^{3 / 2}} \frac{\partial g_{\varphi \varphi}}{\partial r} \\
& +\frac{\tilde{\Omega}\left(-g_{t \varphi}^{2}+f g_{\varphi \varphi} g^{r r}-g_{\varphi \varphi} g_{t \varphi} \tilde{\Omega}\right)\left(g_{t \varphi}^{2}-f g_{\varphi \varphi} g^{r r}+f g_{\varphi \varphi} H g^{r r}+g_{\varphi \varphi} g_{t \varphi} \tilde{\Omega}\right)}{f^{2} g_{\varphi \varphi}^{2} H^{2}\left(g^{r r}\right)^{3 / 2}} \frac{\partial g_{t \varphi}}{\partial r} \\
& +\frac{\left(g_{t \varphi}^{2}-f g_{\varphi \varphi} g^{r r}+f g_{\varphi \varphi} H g^{r r}+g_{\varphi \varphi} g_{t \varphi} \tilde{\Omega}\right)^{2}}{2 f^{2} g_{\varphi \varphi}^{4} H^{2}\left(g^{r r}\right)^{3 / 2}} \\
& \times\left(g_{\varphi \varphi}^{2} g^{r r} \frac{\partial f}{\partial r}+g_{t \varphi}^{2} \frac{\partial g_{\varphi \varphi}}{\partial r}-2 g_{\varphi \varphi} g_{t \varphi} \frac{\partial g_{t \varphi}}{\partial r}+f g_{\varphi \varphi}^{2} \frac{\partial g^{r r}}{\partial r}\right) \\
& K_{t \varphi}^{1}=-\frac{\tilde{\Omega}\left(g_{t \varphi}^{2}-f g_{\varphi \varphi} g^{r r}+g_{\varphi \varphi} g_{t \varphi} \tilde{\Omega}\right)\left(f H g^{r r}+g_{t \varphi} \tilde{\Omega}+g_{\varphi \varphi} \tilde{\Omega}^{2}\right)}{2 f^{2} g_{\varphi \varphi} H^{2}\left(g^{r r}\right)^{3 / 2}} \frac{\partial g_{\varphi \varphi}}{\partial r} \\
& -\frac{\tilde{\Omega}\left(g_{t \varphi}+g_{\varphi \varphi} \tilde{\Omega}\right)\left(g_{t \varphi}^{2}-f g_{\varphi \varphi} g^{r r}+g_{\varphi \varphi} g_{t \varphi} \tilde{\Omega}\right)}{2 f^{2} g_{\varphi \varphi} H^{2}\left(g^{r r}\right)^{3 / 2}} \frac{\partial g_{t \varphi}}{\partial r} \\
& +\frac{\left(-g_{t \varphi}^{2}+f g_{\varphi \varphi} g^{r r}-f g_{\varphi \varphi} H g^{r r}-g_{\varphi \varphi} g_{t \varphi} \tilde{\Omega}\right)\left(f H g^{r r}+g_{t \varphi} \tilde{\Omega}+g_{\varphi \varphi} \tilde{\Omega}^{2}\right)}{2 f^{2} g_{\varphi \varphi} H^{2}\left(g^{r r}\right)^{3 / 2}} \frac{\partial g_{t \varphi}}{\partial r} \\
& +\frac{\left(g_{t \varphi}+g_{\varphi \varphi} \tilde{\Omega}\right)\left(g_{t \varphi}^{2}-f g_{\varphi \varphi} g^{r r}+f g_{\varphi \varphi} H g^{r r}+g_{\varphi \varphi} g_{t \varphi} \tilde{\Omega}\right)}{2 f^{2} g_{\varphi \varphi}^{3} H^{2}\left(g^{r r}\right)^{3 / 2}} \\
& \times\left(g_{\varphi \varphi}^{2} g^{r r} \frac{\partial f}{\partial r}+g_{t \varphi}^{2} \frac{\partial g_{\varphi \varphi}}{\partial r}-2 g_{\varphi \varphi} g_{t \varphi} \frac{\partial g_{t \varphi}}{\partial r}+f g_{\varphi \varphi}^{2} \frac{\partial g^{r r}}{\partial r}\right)
\end{aligned}
$$




$$
\begin{aligned}
K_{\theta \theta}^{1}= & -\frac{\sqrt{g^{r r}}}{2} \frac{\partial g_{\theta \theta}}{\partial r}, \\
K_{\varphi \varphi}^{1}= & -\frac{\left(f H g^{r r}+g_{t \varphi} \tilde{\Omega}+g_{\varphi \varphi} \tilde{\Omega}^{2}\right)^{2}}{f^{2} H^{2}\left(g^{r r}\right)^{3 / 2}}\left[\frac{1}{2} \frac{\partial g_{\varphi \varphi}}{\partial r}+\frac{\left(g_{t \varphi}+g_{\varphi \varphi} \tilde{\Omega}\right)}{\left(f H g^{r r}+g_{t \varphi} \tilde{\Omega}+g_{\varphi \varphi} \tilde{\Omega}^{2}\right)} \frac{\partial g_{t \varphi}}{\partial r}\right] \\
& +\left(g_{t \varphi}+g_{\varphi \varphi} \tilde{\Omega}\right)^{2}\left(g^{r r} \frac{\partial f}{\partial r}+\frac{g_{t \varphi}^{2}}{g_{\varphi \varphi}^{2}} \frac{\partial g_{\varphi \varphi}}{\partial r}-\frac{2 g_{t \varphi}}{g_{\varphi \varphi} \frac{\partial g_{t \varphi}}{\partial r}+f \frac{\partial g^{r r}}{\partial r}}\right) \frac{1}{2 f^{2} H^{2}\left(g^{r r}\right)^{3 / 2}}, \\
K_{t \theta}^{2}= & \frac{\left(-f H g^{r r}\right)^{1 / 2} \tilde{\Omega}\left(-g_{t \varphi}^{2}+f g_{\varphi \varphi} g^{r r}-g_{\varphi \varphi} g_{t \varphi} \tilde{\Omega}\right)}{2 f H g^{r r}\left(-g_{t \varphi}^{2}+f g_{\varphi \varphi} g^{r r}-2 g_{\varphi \varphi} g_{t \varphi} \tilde{\Omega}-g_{\varphi \varphi}^{2} \tilde{\Omega}^{2}\right)}\left(\tilde{\Omega} \frac{\partial g_{\varphi \varphi}}{\partial \theta}+\frac{\partial g_{t \varphi}}{\partial \theta}\right) \\
+ & \left(\left(-f H g^{r r}\right)^{1 / 2}\left(g_{t \varphi}^{2}-f g_{\varphi \varphi} g^{r r}+f g_{\varphi \varphi} H g^{r r}+g_{\varphi \varphi} g_{t \varphi} \tilde{\Omega}\right)\right. \\
\times & \frac{\left(g_{\varphi \varphi}^{2} g^{r r} \frac{\partial f}{\partial \theta}+g_{t \varphi}^{2} \frac{\partial g_{\varphi \varphi}}{\partial \theta}-2 g_{\varphi \varphi} g_{t \varphi} \frac{\partial g_{\varphi}}{\partial \theta}-g_{\varphi \varphi}^{2} \tilde{\Omega} \frac{\partial g_{t \varphi}}{\partial \theta}+f g_{\varphi \varphi}^{2} \frac{\partial g^{r r}}{\partial \theta}\right)}{2 f g_{\varphi \varphi}^{2} H g^{r r}\left(-g_{t \varphi}^{2}+f g_{\varphi \varphi} g^{r r}-2 g_{\varphi \varphi} g_{t \varphi} \tilde{\Omega}-g_{\varphi \varphi}^{2} \tilde{\Omega}^{2}\right)} \\
K_{\varphi \theta}^{2}= & \frac{g_{\varphi \varphi}\left(-f H g^{r r}\right)^{1 / 2}\left(f H g^{r r}+g_{t \varphi} \tilde{\Omega}+g_{\varphi \varphi} \tilde{\Omega}^{2}\right)}{2 f H g^{r r}\left(g_{t \varphi}^{2}-f g_{\varphi \varphi} g^{r r}+2 g_{\varphi \varphi} g_{t \varphi} \tilde{\Omega}+g_{\varphi \varphi}^{2} \tilde{\Omega}^{2}\right)}\left(\tilde{\Omega} \frac{\partial g_{\varphi \varphi}}{\partial \theta}+\frac{\partial g_{t \varphi}}{\partial \theta}\right) \\
& -\left[( - f H g ^ { r r } ) ^ { - 1 / 2 } ( g _ { t \varphi } + g _ { \varphi \varphi } \tilde { \Omega } ) \left(g_{\varphi \varphi}^{2} g^{r r} \frac{\partial f}{\partial \theta}+g_{t \varphi}^{2} \frac{\partial g_{\varphi \varphi}}{\partial \varphi}-2 g_{\varphi \varphi} g_{t \varphi} \frac{\partial g_{t \varphi}}{\partial \theta}\right.\right. \\
& \left.\left.-g_{\varphi \varphi}^{2} \tilde{\Omega} \frac{\partial g_{t \varphi}}{\partial \theta}+f g_{\varphi \varphi}^{2} \frac{\partial g^{r r}}{\partial \theta}\right)\right] \frac{1}{2 g_{\varphi \varphi}\left(-g_{t \varphi}^{2}+f g_{\varphi \varphi} g^{r r}-2 g_{\varphi \varphi} g_{t \varphi} \tilde{\Omega}-g_{\varphi \varphi}^{2} \tilde{\Omega}^{2}\right)}
\end{aligned}
$$

And the contravariant quantities are given by

$$
\begin{aligned}
K_{1}^{t t} & =\left(g_{\varphi \varphi}^{2} g^{r r} \frac{\partial f}{\partial r}-2 g_{\varphi \varphi}^{2} H g^{r r} \frac{\partial f}{\partial r}+g_{\varphi \varphi}^{2} H^{2} g^{r r} \frac{\partial f}{\partial r}+g_{t \varphi}^{2} \frac{\partial g_{\varphi \varphi}}{\partial r}-2 g_{t \varphi}^{2} H \frac{\partial g_{\varphi \varphi}}{\partial r}\right. \\
& -2 g_{\varphi \varphi} g_{t \varphi} H \tilde{\Omega} \frac{\partial g_{\varphi \varphi}}{\partial r}-g_{\varphi \varphi}^{2} \tilde{\Omega}^{2} \frac{\partial g_{\varphi \varphi}}{\partial r}-2 g_{\varphi \varphi} g_{t \varphi} \frac{\partial g_{t \varphi}}{\partial r}(1-H)-2 g_{\varphi \varphi}^{2} \tilde{\Omega} \frac{\partial g_{t \varphi}}{\partial r} \\
& \left.+2 g_{\varphi \varphi}^{2} H \tilde{\Omega} \frac{\partial g_{t \varphi}}{\partial r}+f g_{\varphi \varphi}^{2} \frac{\partial g^{r r}}{\partial r}(1-H)^{2}\right) /\left(2 f^{2} g_{\varphi \varphi}^{2} H^{2}\left(g^{r r}\right)^{3 / 2}\right), \\
K_{1}^{t \varphi} & =\left(g_{\varphi \varphi}^{2} g_{t \varphi} H g^{r r} \frac{\partial f}{\partial r}-g_{\varphi \varphi}^{2} g_{t \varphi} H^{2} g^{r r} \frac{\partial f}{\partial r}+g_{\varphi \varphi}^{3} g^{r r} \tilde{\Omega} \frac{\partial f}{\partial r}(1-H)+g_{t \varphi}^{3} H \frac{\partial g_{\varphi \varphi}}{\partial r}\right. \\
& -f g_{\varphi \varphi} g_{t \varphi} H^{2} g^{r r} \frac{\partial g_{\varphi \varphi}}{\partial r}+g_{\varphi \varphi} g_{t \varphi}^{2} \tilde{\Omega} \frac{\partial g_{\varphi \varphi}}{\partial r}-f g_{\varphi \varphi}^{2} H g^{r r} \tilde{\Omega} \frac{\partial g_{\varphi \varphi}}{\partial r}-g_{\varphi \varphi}^{2} g_{t \varphi} H \tilde{\Omega}^{2} \frac{\partial g_{\varphi \varphi}}{\partial r} \\
& -g_{\varphi \varphi}^{3} \tilde{\Omega}^{3} \frac{\partial g_{\varphi \varphi}}{\partial r}-g_{\varphi \varphi} g_{t \varphi}^{2} H \frac{\partial g_{t \varphi}}{\partial r}-f g_{\varphi \varphi}^{2} H g^{r r} \frac{\partial g_{t \varphi}}{\partial r}+f g_{\varphi \varphi}^{2} H^{2} g^{r r} \frac{\partial g_{t \varphi}}{\partial r} \\
& -2 g_{\varphi \varphi}^{2} g_{t \varphi} \tilde{\Omega} \frac{\partial g_{t \varphi}}{\partial r}-2 g_{\varphi \varphi}^{3} \tilde{\Omega}^{2} \frac{\partial g_{t \varphi}}{\partial r}+g_{\varphi \varphi}^{3} H \tilde{\Omega}^{2} \frac{\partial g_{t \varphi}}{\partial r}+f g_{\varphi \varphi}^{2} g_{t \varphi} H \frac{\partial g^{r r}}{\partial r}(1-H) \\
& \left.+f g_{\varphi \varphi}^{3} \tilde{\Omega} \frac{\partial g^{r r}}{\partial r}-f g_{\varphi \varphi}^{3} H \tilde{\Omega} \frac{\partial g^{r r}}{\partial r}\right) /\left(2 f^{2} g_{\varphi \varphi}^{3} H^{2}\left(g^{r r}\right)^{3 / 2}\right),
\end{aligned}
$$




$$
\begin{aligned}
& K_{1}^{\theta \theta}=-\left(\left(g^{r r}\right)^{1 / 2} \frac{\partial g_{\theta \theta}}{\partial r}\right) /\left(2 g_{\theta \theta}^{2}\right) \\
& K_{1}^{\varphi \varphi}=\left(g_{\varphi \varphi} g_{t \varphi}^{2} H^{2} g^{r r} \frac{\partial f}{\partial r}+2 g_{\varphi \varphi}^{2} g_{t \varphi} H g^{r r} \tilde{\Omega} \frac{\partial f}{\partial r}+g_{\varphi \varphi}^{3} g^{r r} \tilde{\Omega}^{2} \frac{\partial f}{\partial r}+2 f g_{t \varphi}^{2} H^{2} g^{r r} \frac{\partial g_{\varphi \varphi}}{\partial r}\right. \\
& -f^{2} g_{\varphi \varphi} H^{2}\left(g^{r r}\right)^{2} \frac{\partial g_{\varphi \varphi}}{\partial r}+2 g_{t \varphi}^{3} H \tilde{\Omega} \frac{\partial g_{\varphi \varphi}}{\partial r}+g_{\varphi \varphi} g_{t \varphi}^{2} \tilde{\Omega}^{2} \frac{\partial g_{\varphi \varphi}}{\partial r}+2 g_{\varphi \varphi} g_{t \varphi}^{2} H \tilde{\Omega}^{2} \frac{\partial g_{\varphi \varphi}}{\partial r} \\
& -2 f g_{\varphi \varphi}^{2} H g^{r r} \tilde{\Omega}^{2} \frac{\partial g_{\varphi \varphi}}{\partial r}-g_{\varphi \varphi}^{3} \tilde{\Omega}^{4} \frac{\partial g_{\varphi \varphi}}{\partial r}-2 f g_{\varphi \varphi} g_{t \varphi} H^{2} g^{r r} \frac{\partial g_{t \varphi}}{\partial r}-2 g_{\varphi \varphi} g_{t \varphi}^{2} H \tilde{\Omega} \frac{\partial g_{t \varphi}}{\partial r} \\
& -2 f g_{\varphi \varphi}^{2} H g^{r r} \tilde{\Omega} \frac{\partial g_{t \varphi}}{\partial r}-2 g_{\varphi \varphi}^{2} g_{t \varphi} \tilde{\Omega}^{2} \frac{\partial g_{t \varphi}}{\partial r}-2 g_{\varphi \varphi}^{2} g_{t \varphi} H \tilde{\Omega}^{2} \frac{\partial g_{t \varphi}}{\partial r}-2 g_{\varphi \varphi}^{3} \tilde{\Omega}^{3} \frac{\partial g_{t \varphi}}{\partial r} \\
& \left.+f g_{\varphi \varphi} g_{t \varphi}^{2} H^{2} \frac{\partial g^{r r}}{\partial r}+2 f g_{\varphi \varphi}^{2} g_{t \varphi} H \tilde{\Omega} \frac{\partial g^{r r}}{\partial r}+f g_{\varphi \varphi}^{3} \tilde{\Omega}^{2} \frac{\partial g^{r r}}{\partial r}\right) /\left(2 f^{2} g_{\varphi \varphi}^{3} H^{2}\left(g^{r r}\right)^{3 / 2}\right), \\
& K_{2}^{t \theta}=\left[( - f H g ^ { r r } ) ^ { 1 / 2 } \left(2 g_{\varphi \varphi}^{2} g_{t \varphi}^{2} g^{r r} \frac{\partial f}{\partial \theta}-2 g_{\varphi \varphi}^{2} g_{t \varphi}^{2} H g^{r r} \frac{\partial f}{\partial \theta}-f g_{\varphi \varphi}^{3}\left(g^{r r}\right)^{2} \frac{\partial f}{\partial \theta}\right.\right. \\
& +f g_{\varphi \varphi}^{3} H\left(g^{r r}\right)^{2} \frac{\partial f}{\partial \theta}+2 g_{\varphi \varphi}^{3} g_{t \varphi} g^{r r} \tilde{\Omega} \frac{\partial f}{\partial \theta}-2 g_{\varphi \varphi}^{3} g_{t \varphi} H g^{r r} \tilde{\Omega} \frac{\partial f}{\partial \theta}+2 g_{t \varphi}^{4} \frac{\partial g_{\varphi \varphi}}{\partial \theta} \\
& -f g_{\varphi \varphi} g_{t \varphi}^{2} g^{r r} \frac{\partial g_{\varphi \varphi}}{\partial \theta}-f g_{\varphi \varphi} g_{t \varphi}^{2} H g^{r r} \frac{\partial g_{\varphi \varphi}}{\partial \theta}+4 g_{\varphi \varphi} g_{t \varphi}^{3} \tilde{\Omega} \frac{\partial g_{\varphi \varphi}}{\partial \theta} \\
& -(2+H) f g_{\varphi \varphi}^{2} g_{t \varphi} g^{r r} \tilde{\Omega} \frac{\partial g_{\varphi \varphi}}{\partial \theta}+2 g_{\varphi \varphi}^{2} g_{t \varphi}^{2} \tilde{\Omega}^{2} \frac{\partial g_{\varphi \varphi}}{\partial \theta}-f g_{\varphi \varphi}^{3} g^{r r} \tilde{\Omega}^{2} \frac{\partial g_{\varphi \varphi}}{\partial \theta} \\
& -2 g_{\varphi \varphi} g_{t \varphi}^{3} \frac{\partial g_{t \varphi}}{\partial \theta}+f g_{\varphi \varphi}^{2} g_{t \varphi} H g^{r r} \frac{\partial g_{t \varphi}}{\partial \theta}-4 g_{\varphi \varphi}^{2} g_{t \varphi}^{2} \tilde{\Omega} \frac{\partial g_{t \varphi}}{\partial \theta}+f g_{\varphi \varphi}^{3} H g^{r r} \tilde{\Omega} \frac{\partial g_{t \varphi}}{\partial \theta} \\
& -2 g_{\varphi \varphi}^{3} g_{t \varphi} \tilde{\Omega}^{2} \frac{\partial g_{t \varphi}}{\partial \theta}+2 f g_{\varphi \varphi}^{2} g_{t \varphi}^{2} \frac{\partial g^{r r}}{\partial \theta}-2 f g_{\varphi \varphi}^{2} g_{t \varphi}^{2} H \frac{\partial g^{r r}}{\partial \theta}-f^{2} g_{\varphi \varphi}^{3} g^{r r} \frac{\partial g^{r r}}{\partial \theta} \\
& \left.\left.+f^{2} g_{\varphi \varphi}^{3} H g^{r r} \frac{\partial g^{r r}}{\partial \theta}+2(1-H) f g_{\varphi \varphi}^{3} g_{t \varphi} \tilde{\Omega} \frac{\partial g^{r r}}{\partial \theta}\right)\right] /\left(2 f ^ { 2 } g _ { \theta \theta } g _ { \varphi \varphi } ^ { 2 } H ( g ^ { r r } ) ^ { 2 } \left(g_{t \varphi}^{2}\right.\right. \\
& \left.\left.-f g_{\varphi \varphi} g^{r r}+g_{\varphi \varphi} g_{t \varphi} \tilde{\Omega}+g_{\varphi \varphi} g_{t \varphi} \tilde{\Omega}+g_{\varphi \varphi}^{2} \tilde{\Omega} \tilde{\Omega}\right)\right) \\
& K_{2}^{\varphi \theta}=\left[( - f H g ^ { r r } ) ^ { 1 / 2 } \left(-2 g_{\varphi \varphi} g_{t \varphi}^{3} H g^{r r} \frac{\partial f}{\partial \theta}+f g_{\varphi \varphi}^{2} g_{t \varphi} H\left(g^{r r}\right)^{2} \frac{\partial f}{\partial \theta}-2 g_{\varphi \varphi}^{2} g_{t \varphi}^{2} g^{r r} \tilde{\Omega} \frac{\partial f}{\partial \theta}\right.\right. \\
& -2 g_{\varphi \varphi}^{2} g_{t \varphi}^{2} H g^{r r} \tilde{\Omega} \frac{\partial f}{\partial \theta}+f g_{\varphi \varphi}^{3}\left(g^{r r}\right)^{2} \tilde{\Omega} \frac{\partial f}{\partial \theta}-2 g_{\varphi \varphi}^{3} g_{t \varphi} g^{r r} \tilde{\Omega}^{2} \frac{\partial f}{\partial \theta}-3 f g_{t \varphi}^{3} H g^{r r} \frac{\partial g_{\varphi \varphi}}{\partial \theta} \\
& +2 f^{2} g_{\varphi \varphi} g_{t \varphi} H\left(g^{r r}\right)^{2} \frac{\partial g_{\varphi \varphi}}{\partial \theta}-2 g_{t \varphi}^{4} \tilde{\Omega} \frac{\partial g_{\varphi \varphi}}{\partial \theta}+f(1-3 H) g_{\varphi \varphi} g_{t \varphi}^{2} g^{r r} \tilde{\Omega} \frac{\partial g_{\varphi \varphi}}{\partial \theta} \\
& +f^{2} g_{\varphi \varphi}^{2} H\left(g^{r r}\right)^{2} \tilde{\Omega} \frac{\partial g_{\varphi \varphi}}{\partial \theta}-4 g_{\varphi \varphi} g_{t \varphi}^{3} \tilde{\Omega}^{2} \frac{\partial g_{\varphi \varphi}}{\partial \theta}+2 f g_{\varphi \varphi}^{2} g_{t \varphi} g^{r r} \tilde{\Omega}^{2} \frac{\partial g_{\varphi \varphi}}{\partial \theta} \\
& +f g_{\varphi \varphi}^{3} g^{r r} \tilde{\Omega}^{3} \frac{\partial g_{\varphi \varphi}}{\partial \theta}+3 f g_{\varphi \varphi} g_{t \varphi}^{2} H g^{r r} \frac{\partial g_{t \varphi}}{\partial \theta}-f^{2} g_{\varphi \varphi}^{2} H\left(g^{r r}\right)^{2} \frac{\partial g_{t \varphi}}{\partial \theta}+2 g_{\varphi \varphi} g_{t \varphi}^{3} \tilde{\Omega} \frac{\partial g_{t \varphi}}{\partial \theta} \\
& +3 f g_{\varphi \varphi}^{2} g_{t \varphi} H g^{r r} \tilde{\Omega} \frac{\partial g_{t \varphi}}{\partial \theta}+4 g_{\varphi \varphi}^{2} g_{t \varphi}^{2} \tilde{\Omega}^{2} \frac{\partial g_{t \varphi}}{\partial \theta}+2 g_{\varphi \varphi}^{3} g_{t \varphi} \tilde{\Omega}^{3} \frac{\partial g_{t \varphi}}{\partial \theta}-2 f g_{\varphi \varphi} g_{t \varphi}^{3} H \frac{\partial g^{r r}}{\partial \theta} \\
& +f^{2} g_{\varphi \varphi}^{2} g_{t \varphi} H g^{r r} \frac{\partial g^{r r}}{\partial \theta}-2 f g_{\varphi \varphi}^{2} g_{t \varphi}^{2} \tilde{\Omega} \frac{\partial g^{r r}}{\partial \theta}-2 f g_{\varphi \varphi}^{2} g_{t \varphi}^{2} H \tilde{\Omega} \frac{\partial g^{r r}}{\partial \theta}+f^{2} g_{\varphi \varphi}^{3} g^{r r} \tilde{\Omega} \frac{\partial g^{r r}}{\partial \theta} \\
& \left.\left.-2 f g_{\varphi \varphi}^{3} g_{t \varphi} \tilde{\Omega}^{2} \frac{\partial g^{r r}}{\partial \theta}-2 g_{\varphi \varphi}^{2} g_{t \varphi}^{2} \tilde{\Omega}^{3} \frac{\partial g_{\varphi \varphi}}{\partial \theta}\right)\right] /\left(2 f ^ { 2 } g _ { \theta \theta } g _ { \varphi \varphi } ^ { 2 } H ( g ^ { r r } ) ^ { 2 } \left(-g_{t \varphi}^{2}-g_{\varphi \varphi}^{2} \tilde{\Omega}^{2}\right.\right.
\end{aligned}
$$




$$
\left.-g_{\varphi \varphi} g_{t \varphi} \tilde{\Omega}+f g_{\varphi \varphi} g^{r r}\right)
$$

The trace of the extrinsic curvature $k^{a}=g^{\mu \nu} k_{\mu \nu}^{a}$ are

$$
\begin{aligned}
K^{1} & =\frac{1}{2 f^{2} g_{\theta \theta} g_{\varphi \varphi}^{3} H^{2}\left(g^{r r}\right)^{3 / 2}}\left(g_{\theta \theta} g_{\varphi \varphi}^{2} g_{t \varphi}^{2} g^{r r} \frac{\partial f}{\partial r}-f(1-H)^{2} g_{\theta \theta} g_{\varphi \varphi}^{3}\left(g^{r r}\right)^{2} \frac{\partial f}{\partial r}\right. \\
& +2 g_{\theta \theta} g_{\varphi \varphi}^{3} g_{t \varphi} g^{r r} \tilde{\Omega} \frac{\partial f}{\partial r}+g_{\theta \theta} g_{\varphi \varphi}^{4} g^{r r} \tilde{\Omega}^{2} \frac{\partial f}{\partial r}-f^{2} g_{\varphi \varphi}^{3} H^{2}\left(g^{r r}\right)^{2} \frac{\partial g_{\theta \theta}}{\partial r} \\
& +g_{\theta \theta} g_{t \varphi}^{4} \frac{\partial g_{\varphi \varphi}}{\partial r}-f(1-2 H) g_{\theta \theta} g_{\varphi \varphi} g_{t \varphi}^{2} g^{r r} \frac{\partial g_{\varphi \varphi}}{\partial r}-f^{2} g_{\theta \theta} g_{\varphi \varphi}^{2} H^{2}\left(g^{r r}\right)^{2} \frac{\partial g_{\varphi \varphi}}{\partial r} \\
& +2 g_{\theta \theta} g_{\varphi \varphi} g_{t \varphi}^{3} \tilde{\Omega} \frac{\partial g_{\varphi \varphi}}{\partial r}+f(1-2 H) g_{\theta \theta} g_{\varphi \varphi}^{3} g^{r r} \tilde{\Omega}^{2} \frac{\partial g_{\varphi \varphi}}{\partial r}-2 g_{\theta \theta} g_{\varphi \varphi}^{3} g_{t \varphi} \tilde{\Omega}^{3} \frac{\partial g_{\varphi \varphi}}{\partial r} \\
& -g_{\theta \theta} g_{\varphi \varphi}^{4} \tilde{\Omega}^{4} \frac{\partial g_{\varphi \varphi}}{\partial r}-2 g_{\theta \theta} g_{\varphi \varphi} g_{t \varphi}^{3} \frac{\partial g_{t \varphi}}{\partial r}+2 f(1-2 H) g_{\theta \theta} g_{\varphi \varphi}^{2} g_{t \varphi} g^{r r} \frac{\partial g_{t \varphi}}{\partial r} \\
& -6 g_{\theta \theta} g_{\varphi \varphi}^{2} g_{t \varphi}^{2} \tilde{\Omega} \frac{\partial g_{t \varphi}}{\partial r}+2 f(1-2 H) g_{\theta \theta} g_{\varphi \varphi}^{3} g^{r r} \tilde{\Omega} \frac{\partial g_{t \varphi}}{\partial r}-6 g_{\theta \theta} g_{\varphi \varphi}^{3} g_{t \varphi} \tilde{\Omega}^{2} \frac{\partial g_{t \varphi}}{\partial r} \\
& -2 g_{\theta \theta} g_{\varphi \varphi}^{4} \tilde{\Omega}^{3} \frac{\partial g_{t \varphi}}{\partial r}+f g_{\theta \theta} g_{\varphi \varphi}^{2} g_{t \varphi}^{2} \frac{\partial g^{r r}}{\partial r}-f^{2} g_{\theta \theta} g_{\varphi \varphi}^{3} g^{r r} \frac{\partial g^{r r}}{\partial r}+2 f^{2} g_{\theta \theta} g_{\varphi \varphi}^{3} H g^{r r} \frac{\partial g^{r r}}{\partial r} \\
& \left.-f^{2} g_{\theta \theta} g_{\varphi \varphi}^{3} H^{2} g^{r r} \frac{\partial g^{r r}}{\partial r}+2 f g_{\theta \theta} g_{\varphi \varphi}^{3} g_{t \varphi} \tilde{\Omega}^{\frac{\partial g}{r r}} \frac{\partial g^{r r}}{\partial r}+f g_{\theta \theta} g_{\varphi \varphi}^{4} \tilde{\Omega}^{2} \frac{\partial g^{r r}}{\partial r}\right), \\
K^{2} & =0 .
\end{aligned}
$$

All quantities listed by Eqs. (B3), (B44), (B5), (B5), and (B6) are equal to zero ( they are proportional to $\sqrt{g^{r r}}$ near the event horizon) on the event horizon. Consequently, on the event horizon, we obtain

$$
\begin{aligned}
K^{a} K^{a} & =0, \\
\operatorname{tr}(K . K) & =K_{\mu \nu}^{a} K_{a}^{\mu \nu}=0 .
\end{aligned}
$$

which show that the quadratic combinations of the extrinsic curvature are zero on the event horizon when we use the vectors $(\mathrm{A2})$. The calculation for the Kerr-Newman and EinsteinMaxwell dilaton-axion black holes in Refs. [11] [33] supported the result. 


\section{REFERENCES}

[1] V. P. Frolov and D. V. Fursaev, Class. Quantum Grav. 15, 2041 (1998).

[2] S. N. Solodukhin, Phys. Rev. D 51, 618 (1995).

[3] D. V. Fursaev, Class. Quantum Grav. 11, 1431 (1994 ).

[4] G. Cognola, K. Kirsten, and L. Vanzo, Phys. Rev. D 49, 1029 (1994 ).

[5] S. N. Solodukhin, Phys. Rev. D 51, 609 (1995).

[6] S. N. Solodukhin, Phys. Rev. D 52, 7046 (1995).

[7] A. O. Barvinsky and S. N. Solodukhin, Nucl. Phys. B 479, 305 (1996).

[8] D. V. Fursaev, Phys. Lett. B 334, 53 (1994).

[9] D. V. Fursaev, Mod. Phys. Lett. A10, 649 (1995).

[10] D. V. Fursaev and S. N. Solodukhin, Phys. Lett. B 365, 51 (1996).

[11] R. B. Mann and S. N. Solodukhin, Phys. Rev. D 54, 3932 (1996).

[12] G. 't. Hooft, Nucl. Phys. B 256, 727 (1985).

[13] S. N. Solodukhin, Phys. Rev. D 56, 4968 (1997).

[14] J. G. Demers, R. Lafrance, and R.C. Myers, Phys. Rev. D 52, 2245 (1995).

[15] A. Ghosh and P. Mitra, Phys. Rev. Lett. 73, 2521 (1994).

[16] M.H. Lee and J. K. Kim, Phys. Lett. A 212, 323 (1996).

[17] R. B. Mann, L. Tarasov, and A. Zelnikov, Class. Quantum Grav. 9, 1487 (1992).

[18] A. Romeo, Class. Quantum Grav. 13, 2797 (1996).

[19] S. P. Kim et al Phys. Rev. D 55, 2159 (1997).

[20] G. Cognola and P. Lecca, Phys. Rev. D 57, 2159 (1998).

[21] M.H. Lee and J. K. Kim, Phys. Rev. D 54, 3904 (1996).

[22] M.H. Lee, H. C. Kim, and J. K. Kim, Phys. Lett. B 388, 487 (1996).

[23] J. Ho, W. T. Kim and Y. J. Park, "Entropy in the Kerr-Newman black hole". grqc/9704032, 1997.

[24] S. W. Kim, W. T. Kim, Y. J. Park, and H. Shin, Phys. Lett. B 392, 311 (1997).

[25] F. Belgiorno and S. Liberati, Phys. Rev. D 53, 3172 (1996).

[26] Jiliang Jing, Int. J. Theor. Phys. 371441 (1998).

[27] Jiliang Jing, Chin. Phys. Lett. 14, 495 (1997).

[28] S. N. Solodukhin, Phys. Rev. D 54, 3900 (1996).

[29] V. P. Frolov, D. V. Fursaev, and A. I. Zelnikov, Phys. Lett. B 382, 220 (1996).

[30] V. P. Frolov, D. V. Fursaev, and A. I. Zelnikov, Phys. Rev. D 54, 2711 (1996).

[31] D. V. Fursaev, Euclidean and Canonical Formulations of Statistical Mechanics in the Presence of Killing Horizon, hep-th/9709213.

[32] Jiliang Jing and Mu-Lin Yan, gr-qc/9904001; Phys. Rev. D60, (1999) 084015.

[33] Jiliang Jing, Nucl. Phys.B 476, 548 (1996).

[34] N. D. Birrell and P.C.W.Devies, Quantum Fields in Curved Space (Cambridge University Press, Cambridge, England,1982).

[35] D. V. Fursaev and S. N. Solodukhin, Phys. Rev. D 52, 2133 (1995).

[36] R. M. Wald, Phys. Rev. D 48, R3427 (1993).

[37] V. Iyer and R. M. Wald, Phys. Rev. D 50, 846 (1994).

[38] T. A. Jacobson, G. Kang, and R. C. Myers, Phys. Rev. D 49, 6587 (1994).

[39] F. Larsen and F. Wilczek, Nucl. Phys. B 458, 249 (1996).

[40] D. Kabat, Nucl. Phys. Nucl. Phys. B 453, 281 (1995). 